\title{
Editorial
}

\section{Additive Manufacturing of Biomaterials, Tissues, and Organs}

\begin{abstract}
The introduction of additive manufacturing (AM), often referred to as three-dimensional (3D) printing, has initiated what some believe to be a manufacturing revolution, and has expedited the development of the field of biofabrication. Moreover, recent advances in AM have facilitated further development of patient-specific healthcare solutions. Customization of many healthcare products and services, such as implants, drug delivery devices, medical instruments, prosthetics, and in vitro models, would have been extremely challenging - if not impossible - without AM technologies. The current special issue of the Annals of Biomedical Engineering presents the latest trends in application of AM techniques to healthcare-related areas of research. As a prelude to this special issue, we review here the most important areas of biomedical research and clinical practice that have benefited from recent developments in additive manufacturing techniques. This editorial, therefore, aims to sketch the research landscape within which the other contributions of the special issue can be better understood and positioned. In what follows, we briefly review the application of additive manufacturing techniques in studies addressing biomaterials, (re)generation of tissues and organs, disease models, drug delivery systems, implants, medical instruments, prosthetics, orthotics, and AM objects used for medical visualization and communication.
\end{abstract}

Keywords-Bioprinting, Biofabrication, Biomaterials, Drug delivery, Medical devices, Tissue regeneration.

\section{INTRODUCTION}

Additive manufacturing (AM), also known as 3D printing, has emerged during recent years as a flexible and powerful technique for advanced manufacturing in healthcare. Even though the underlying technology has been in development for more than two decades, the level of maturity and perfection required for real-world applications has been achieved only recently. Most importantly, a wide range of biomedical materials can now be processed using additive manufacturing techniques with increasing accuracy. Moreover, a number of AM processes and the resulting products have already been approved by regulatory bodies for (routine) clini-

${ }^{1}$ Food and Drug Administration (FDA), Technical Considerations for Additive Manufactured Devices-Draft Guidance for Industry and Food and Drug Administration Staff, Issued on May 10, 2016. cal use, and a draft version of FDA guidance for additively manufactured devices has already been published. ${ }^{1}$ At the same time, AM technology has been applied for (re)generation of living tissue structures that could be applied as regenerative implants and disease models. This field of "biofabrication" 28 is developing exponentially, underscoring the potential of applying AM in healthcare. Some other areas, such as pharmacology, oncology, surgery, and rehabilitation have also provided interesting clinical and research applications for additive manufacturing.

The current special issue aims to review and showcase some of the most promising trends in application of AM to

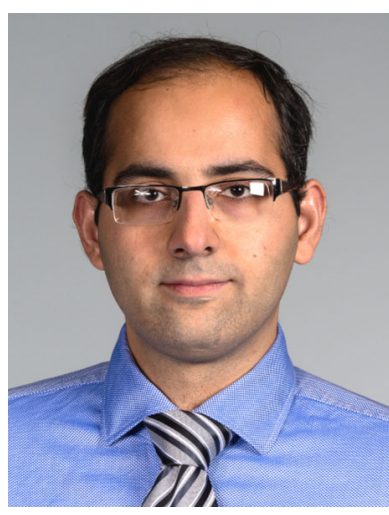

Amir A. Zadpoor

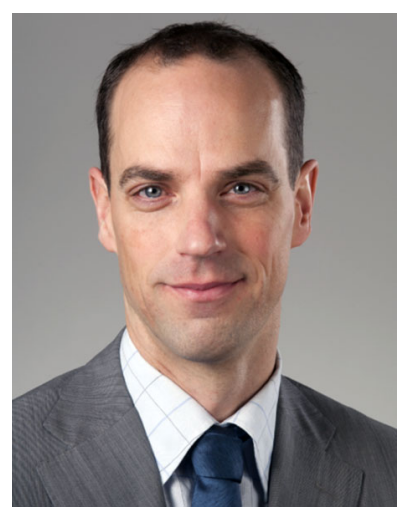

Jos Malda healthcare. This review and the research articles presented here cover a wide range of applications, ranging from cardiovascular ${ }^{19}$ to orthopedic, ${ }^{9,10,58}$ craniofacial, ${ }^{50}$ and drug screening. ${ }^{71}$ As a prelude to this special issue, we decided to write an extended editorial and briefly review the most important trends in application of AM to healthcare, thereby setting the stage for what appears in the rest of the issue. In that sense, this editorial might be seen as a "review of reviews," where we do not try to engage in the details of various areas of research but rather to sketch the bigger picture through clear examples, reference to the papers appearing in this special 
issue, as well as to the other essential literature. In particular, we have not tried to review the details of AM techniques (Fig. 1), as these can be found in some of the excellent review papers appearing in this issue; see, e.g., Refs. 32, 48, 53, 79. Instead, the areas where AM could improve the quality of healthcare are reviewed in the following sections of this editorial, which are organized in order of perceived impact.

\section{ADDITIVE MANUFACTURING OF BIOMATERIALS}

The form freedom offered by AM techniques provides many opportunities for fabrication of biomaterial constructs with complex and precisely controlled external and internal shape. Although the external or macroscale shape of biomaterial structures is important, AM offers the additional opportunity to also control the internal shape or microarchitecture of the generated structures (Fig. 2), which may positively influence tissue regeneration and integration. Moreover, the internal microarchitecture will affect the physical, mechanical, and biological properties of porous biomaterials; ${ }^{75}$ For example, the static mechanical properties, ${ }^{2,8,60}$ fatigue behavior, ${ }^{4}$ and permeability ${ }^{69}$ of porous biomaterials have been shown to be functions of their geometrical parameters. Other geometrical features such as the sign and intensity of curvature have been shown to regulate the rate of tissue regeneration. ${ }^{22,57,75} \mathrm{AM}$ techniques make it possible to use almost any design of microarchitecture to achieve a desired set of physical, mechanical, and biological properties. Furthermore, full interconnectivity in the porous space of biomaterials can be achieved. By rationally designing the microarchitecture, unusual mechanical properties, such as negative Poisson's ratio or independently varying, i.e., decoupled, porosity and mechanical properties, can be achieved as well. ${ }^{76}$ Such rational design of biomaterial microarchitecture is something that fits within the larger context of mechanical metamaterials and has received increasing attention recently ${ }^{73}$ Furthermore, the microarchitecture of biodegradable biomaterials influences their degradation profile and the resulting tissue regeneration performance of (highly) porous biomaterials. ${ }^{13,14,78}$ Rational design of microarchitecture and subsequent AM could, therefore, also be used for adjustment of the biodegradation profile of biomaterials.

Various categories of AM techniques (Fig. 1) have been used for processing a wide range of polymeric, metallic, and ceramic biomaterials. As far as polymeric materials are concerned, AM techniques based on vat polymerization, such as stereolithography (SLA), those based on material extrusion techniques such as fused deposition modeling (FDM), those based on powder bed fusion technologies such as selective laser sintering (SLS), and material jetting alternatives, such as inkjet printing, are commonly used (Fig. 1). The most widely used techniques for processing metallic biomaterials are currently based on powder bed fusion, such as selective laser melting (SLM) and electron beam melting (EBM) (Fig. 2). A large number of studies using AM techniques for processing of ceramicbased biomaterials applied binder jetting, material extrusion, powder bed fusion, or vat polymerization. ${ }^{1,64}$ However, indirect $\mathrm{AM}^{32}$ is another, particularly interesting approach, where biomaterials are not made through direct AM but are fabricated through a medium that is additively manufactured; For example, the negative of an intended biomaterial structure may be additively manufactured to allow for casting of the desired biomaterial. Direct and indirect methods can also be combined to enable fabrication of more complex biomaterial components.

There are two major challenges that need to be addressed to utilize the maximum potential of AM techniques for improving the performance of biomaterials. First, the optimal microarchitecture for the performance of each biomaterial is often unclear. Analytical and multiphysics computational modeling techniques need to be used to determine the best microarchitecture for any specific application. Ideally, all relevant mechanical, physical, and biological properties of the biomaterial should be considered simultaneously when designing the microarchitecture. Secondly, there is still limited availability of materials that are compatible with AM processes. Traditionally used biomaterials can often not be processed with AM techniques, whilst the best-performing materials in AM machines, in terms of accuracy and functionality, are not biocompatible or do not exhibit the required biodegradation behavior. It is, therefore, essential not only to improve the arsenal of available biomaterials, but also to adapt current AM technologies to better process the best available biomaterials. In view of this, developments in both AM materials and systems is required to utilize the potential of AM to its full extent.

\section{TISSUE AND ORGAN EQUIVALENTS}

Biofabrication encompasses the automated generation of tissue constructs by means of bioprinting, bioassembly, and subsequent maturation. As such, it offers the opportunity to generate constructs that more closely match the composition and structure of native tissues. An important difference between biofabrica- 
tion (Fig. 3) and other types of AM is the incorporation of cells in the printed biomaterial, ${ }^{41,44,59}$ which together with the cells is referred to as bioink. The most widely applied AM techniques for bioprinting with bioinks are based on laser-induced forward transfer (LiFT), inkjet printing, and robotic dispensing. ${ }^{41}$ The advantages, disadvantages, and limitations of each of these techniques are further reviewed in a number of contributions to this special issue. ${ }^{19,39,53,71}$ The printed combination of biomaterials, biomolecules, and cells is supposed to gradually mature into the desired tissue. The incorporated biomaterial ideally provides the required initial mechanical support, structural support for mass and gas transfer, and physical cues for activating the appropriate mechanotransductory pathways. At the same time, the biomolecules incorporated into the bioink provide the required biological cues to guide the tissue regeneration process. Multiple bioinks and cell types can be distributed within the same tissue construct to best guide the tissue generation process and to enable regeneration of more complex tissue structures. Ad- vanced imaging can assist with quantification of the shape of defects in the tissue/organ and potentially also its specific composition. The acquired images could be further processed to obtain a computer-aided design (CAD) file describing the exact geometry of the desired patient-specific tissue/organ construct. The patientspecific aspect is further underscored by the potential to use autologous cell sources, minimizing the chance of rejection of the generated tissue/organ. Biofabrication is currently being explored as an approach for generation of various types of tissue constructs, including cartilage, ${ }^{58}$ bone, ${ }^{39}$ skin, ${ }^{39}$ periodontal tissues, ${ }^{12}$ different types of vascularized tissues, ${ }^{56}$ and cardiovascular tissues. ${ }^{19}$ In addition to generating tissue constructs for replacement or repair of damaged tissues, bioprinted tissues could also be used in vitro as tissue analogies in toxicity and disease models ${ }^{72}$ or for (patient-specific) drug screening, ${ }^{71}$ potentially decreasing the need for animal experiments.

Nevertheless, it still remains a challenge to ensure that the generated bioprinted tissue structures properly match the structure and properties of the native tissue.

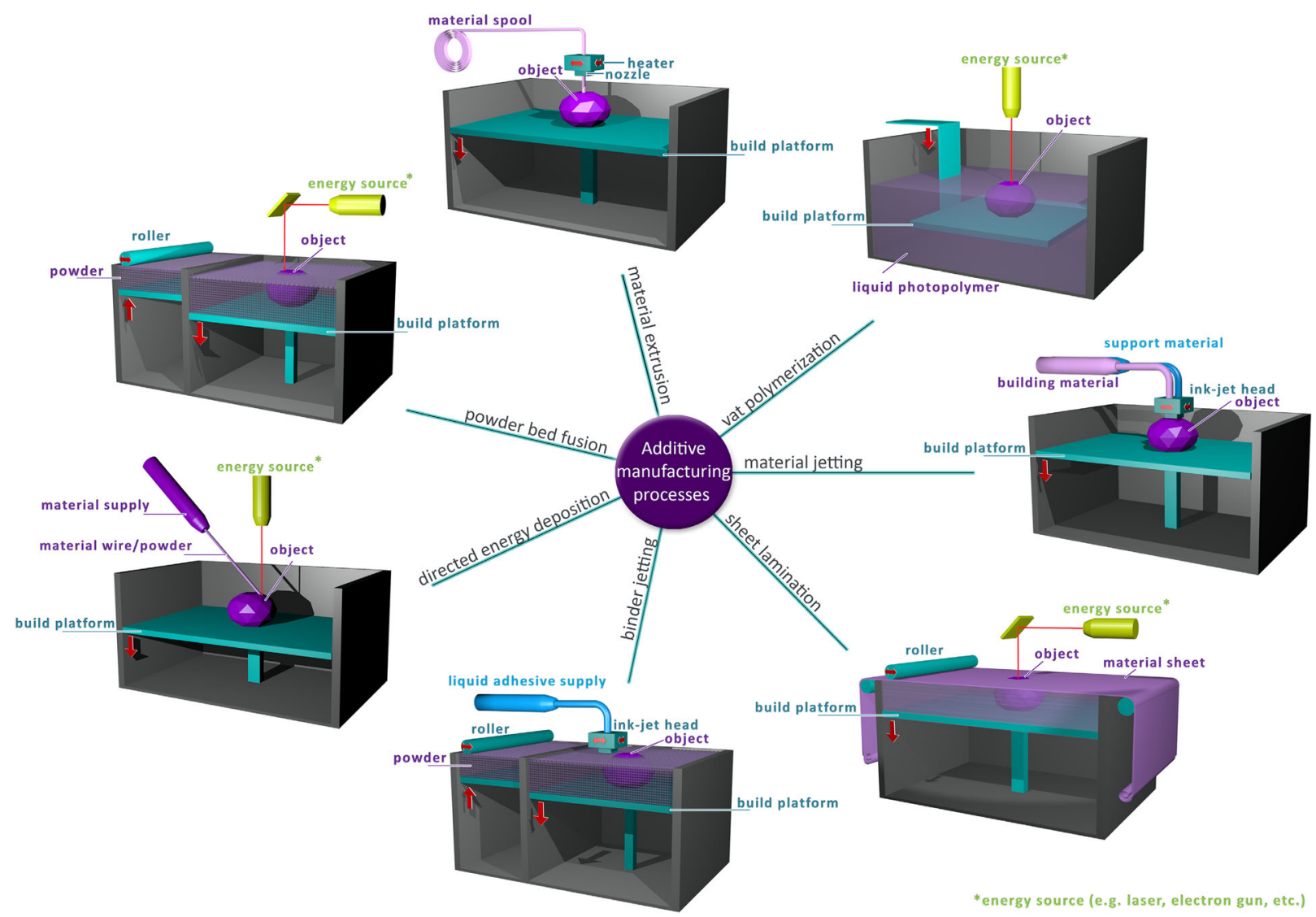

FIGURE 1. Different categories of additive manufacturing technologies according to the terminology proposed by ISO/ ASTM52900:2015. 

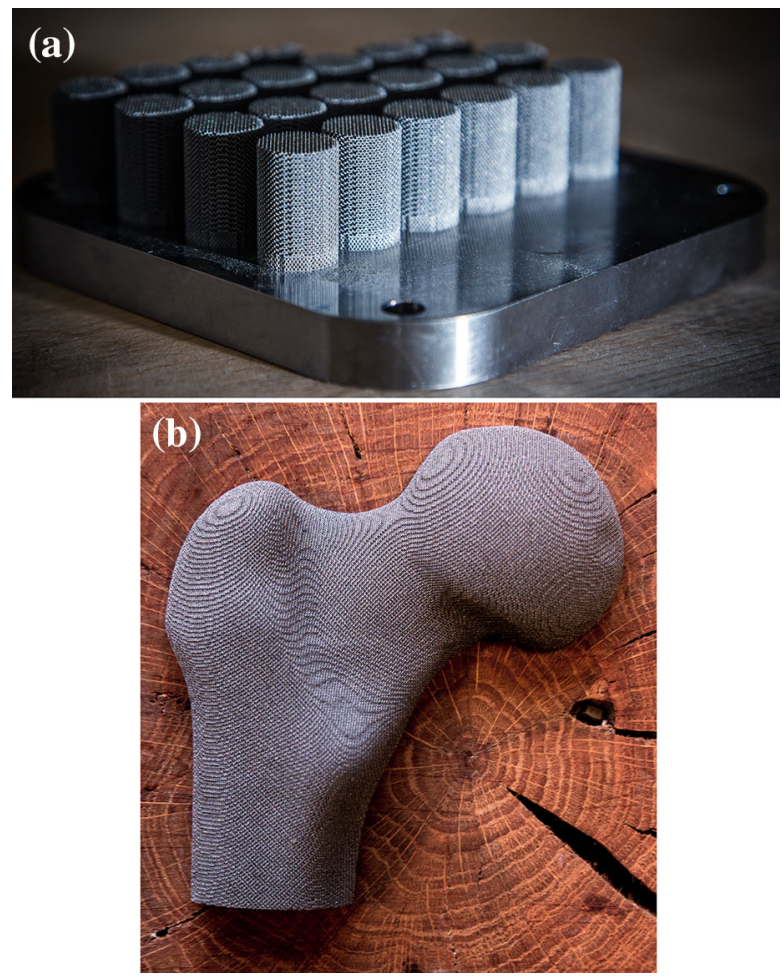

FIGURE 2. Additively manufactured porous titanium in the shape of cylinders (a) and the femur (b) fabricated using selective laser melting from Ti-6Al-4V at the Additive Manufacturing Laboratory, TU Delft (Medical Delta (C) de Beeldredacteur).

A current limitation is the limited availability of bioinks that possess appropriate physical properties for the printing process and simultaneously provide a suitable niche for the cells to differentiate towards the desired lineage. Different classes of hydrogels have been employed as parts of bioink systems used in tissue/organ bioprinting. ${ }^{41,43,45,55,62}$

A promising approach to simultaneously comply with the numerous requirements that AM techniques and bioinks must satisfy to guarantee optimal tissue quality and maximum tissue complexity is to combine various AM technologies and bioinks to benefit from the best aspects of different approaches. Recent application of this pragmatic approach has produced some promising results. ${ }^{36}$

\section{DRUGS AND DRUG DELIVERY}

Various techniques for drug administration and delivery devices including solid dosage forms, ${ }^{26}$ implantable drug delivery vehicles, ${ }^{23,33}$ and topical drug delivery systems ${ }^{25}$ could benefit from what AM has to offer. The recent approval of an AM drug product by the FDA in August $2015^{49}$ marked the beginning of an era where more additively manufactured drugs are expected to enter routine clinical use. In traditional drug delivery research, the main focus is on controlling the release profile through various approaches, among which the most important is development of new biomaterials with distinct, controllable, and predictable release profiles. AM offers an alternative approach for development of new drug delivery systems with tailorable release profiles by adjusting the $3 \mathrm{D}$ shape ${ }^{26}$ and microarchitecture of the drug delivery system, as well as by varying the spatial distribution of active agents ${ }^{27}$ (Fig. 4). Moreover, multiple drugs could be integrated into a single drug delivery system with the possibility of precisely controlling the release profiles of individual drugs (Fig. 4). Furthermore, AM allows for on-demand manufacturing of drug delivery systems, ${ }^{49}$ which is particularly useful for unstable drugs with limited shelf life. ${ }^{49}$ The shape and dose could also be adjusted relatively easily. AM techniques based on binder jetting, material extrusion, ${ }^{18}$ and material jetting could be used for fabrication of drug delivery systems ${ }^{49}$ (Fig. 1). Among the different categories of drug delivery systems, solid dosage forms have received the most attention given their relatively easy route to clinical use and huge potential for commercialization. The effects of the above-mentioned design parameters on the release profiles of drug delivery systems in general and solid dosage forms in particular have not yet been fully understood and require further research.

Computational modeling can aid prediction of release profiles from various drug delivery systems ${ }^{6,31,40}$ and may be of particular value for drug delivery systems based on AM structures. These computational models will provide insights into the effects of the geometrical design, microarchitecture, and spatial distributions of active and passive agents on the release profiles. The combination of AM techniques and computational models for achieving desired release profiles is a relatively unexplored area of research and is suggested to be an important area for future research.

\section{IMPLANTS}

AM has added a new dimension to the design and manufacturing of implants in general, and patientspecific implants in particular. Patient-specific implants, ${ }^{24,46,47}$ where the implant is designed to fit the anatomy or other requirements of a single patient, are one of the prime areas for routine clinical application of AM techniques. Recent advances close the loop in the pipeline that goes from image acquisition to image processing, implant design, and implant manufactur- 

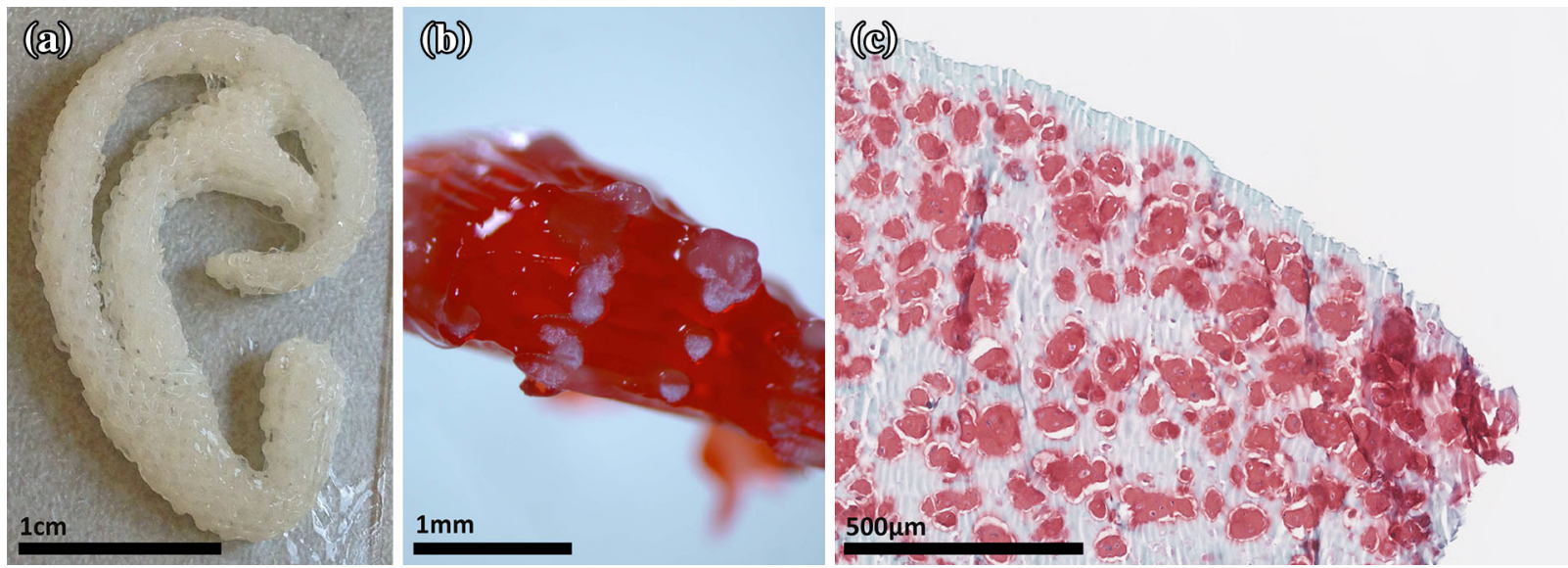

FIGURE 3. Biofabricated auricular implant: (a) macroscopic appearance of a fiber-reinforced biofabricated auricular construct based on gelatin methacryloyl hydrogel and polycaprolactone fibers, (b) magnified view of reinforcing fibers (white) in the hydrogel (red), (c) Safranin 0 staining (stains proteoglycans red) of a histological section of a gelatin methacryloyl hydrogel construct after 6 weeks in vivo (subcutaneous mouse model) (Utrecht Biofabrication Facility, courtesy of Iris Otto, University Medical Center Utrecht).

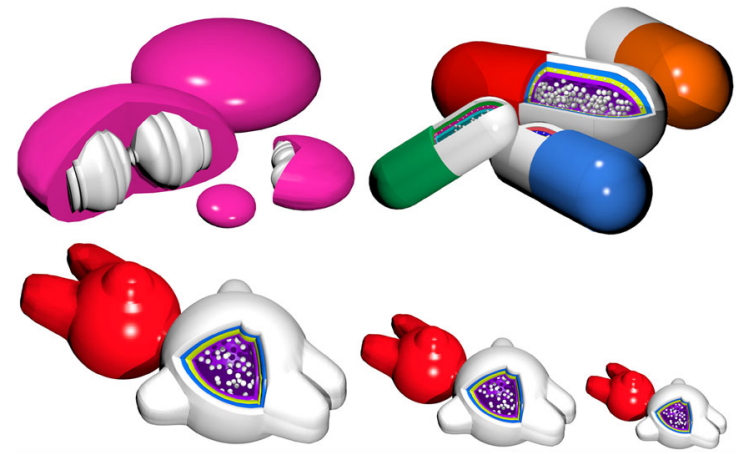

FIGURE 4. Additive manufacturing techniques could be used to (1) achieve complex distribution of several components in solid dosage forms, (2) develop drug products for specific patient groups, e.g., children, and (3) adjust the dosage of drug products.

ing, as the entire process can now be streamlined through CAD systems that integrate some or all of the required steps. The free-form nature of AM processes enables implants with anatomically complex geometries to be manufactured quickly, reliably, and costeffectively. Companies that integrate the various aspects required for patient-specific AM are already active in the market, and their implants are already used in the clinic.

In addition to enabling production of patientspecific implants, AM allows for incorporation of complex geometrical features not only in patientspecific implants but also in generic implants; For example, additively manufactured implants could incorporate rationally designed lattice structures into their design (Fig. 5) to adjust the mechanical properties of the implants, thereby preventing the stressshielding phenomenon. Moreover, the large pore spaces provided by these lattice structures facilitate tissue ingrowth and osseointegration. These structures also provide pore spaces, which could be used for drug delivery purposes, e.g., to facilitate tissue regeneration or combat infection. ${ }^{70}$ Finally, lattice structures have huge adjustable surface areas that could be biofunctionalized $^{68}$ to achieve improved tissue regeneration performance $^{3,17}$ and antibacterial behavior. .,67 $^{2,}$

Ultimately, the design of hybrid implants could integrate solid volumes with various types of lattice structure (Fig. 5). This allows for optimal distribution of mechanical properties within the implant, providing sufficient mechanical support in areas where mechanical stress is greatest but allowing for incorporation of porous structures in areas where stresses are lower, tissue unloading should be avoided, or bone ingrowth is essential, such as the surface of the anchoring parts of the implant. Functionally graded geometries (Fig. 6) could also be realized using AM techniques such that, for example, the porosity of the lattice structure gradually decreases from the implant surface, which is in contact with tissue and could benefit from tissue ingrowth, to the center of the implant, which may need to be stronger to carry mechanical loads.

Metals are the materials most commonly used for AM of functional and load-bearing implants. Powder bed fusion processes including selective laser melting (SLM) and electron beam melting (EBM) are often used for this purpose.

Streamlined design and digital manufacturing of patient-specific implants and incorporation of complex geometrical features into the design of generic implants, as well as evaluation of the actual clinical performance of patient-specific implants and implants incorporating features such as hybrid design and 
functionally graded microarchitecture, are areas that require further research. In particular, sound design principles and computational platforms need to be developed to facilitate the design of the macroscale shape and microarchitecture of implants.

\section{MEDICAL INSTRUMENTS}

Similar to the case of implants, one of the unique applications of AM is fabrication of patient-specific instruments. An important example of patient-specific instruments are surgical guides,${ }^{38}$ which could increase surgical accuracy. Moreover, they could shorten operating times, thereby also reducing the chance of surgery-related complications such as infections. In addition to numerous surgical procedures, where use of such guides could be of great value, patient-specific instruments could be combined with patient-specific implants to facilitate their positioning.

Medical devices could benefit from the form flexibility offered by AM as well. Medical devices with complex shape and advanced functionality could be manufactured to achieve things that cannot be
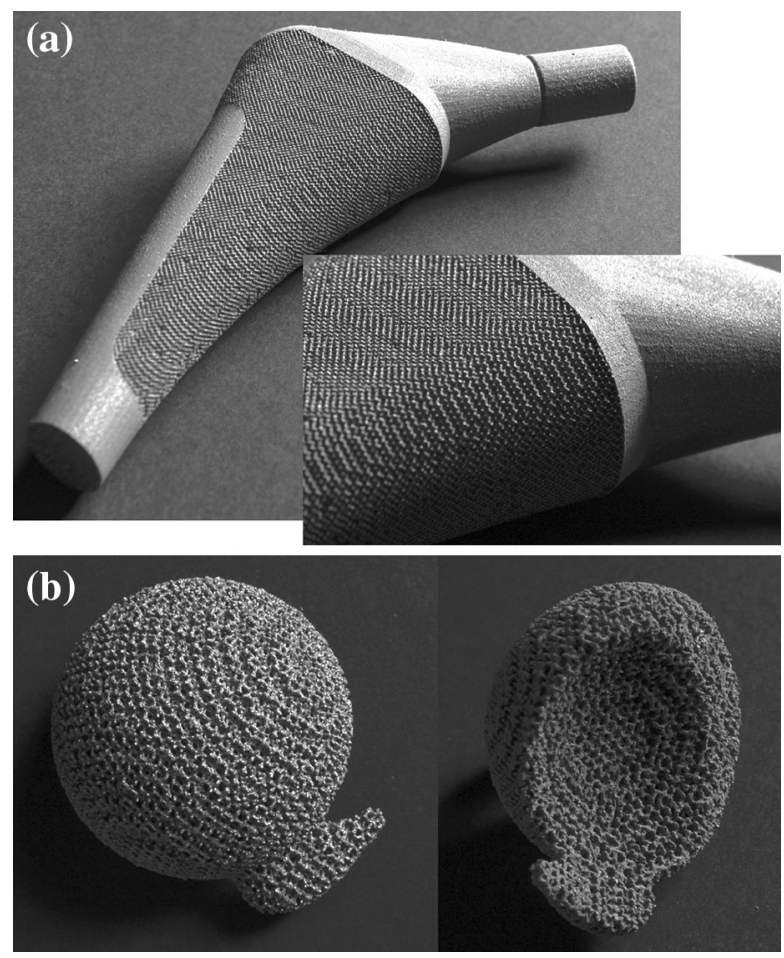

FIGURE 5. (a) Demonstrative hybrid implant that combines solid parts with rationally designed porous parts in a single piece manufactured using a single-step additive manufacturing process. (b) Part of a patient-specific implant designed based on computed tomography (CT) images and manufactured using selective laser melting (Additive Manufacturing Laboratory, TU Delft). achieved with conventionally manufactured instruments. However, manufacturing of high-end medical instruments, particularly steerable instruments, ${ }^{34,35}$ (Fig. 7) often requires very high accuracy and precise control of tolerances. Recent advances in AM techniques have enabled the achievement of extremely high accuracies in manufacturing of medical devices. In this regard, high-resolution AM techniques ${ }^{66}$ such as stereolithography ${ }^{42}$ and two-photon polymerization $^{21,51,54}$ that could achieve accuracies in the range of a few microns, or sometimes submicron, play an important role. However, such applications of superhigh-resolution AM have not been fully explored yet. It is important to realize that biocompatibility requirements are much less stringent for medical instruments as compared with implants and biomaterials that are supposed to dwell in the human body for a long time and require the highest levels of biocompatibility. These less stringent biocompatibility requirements make it possible to use a wider range of materials, thereby increasing the chance of meeting the accuracies and tolerances required for fabrication of (steerable) medical instruments. At the low end, AM could enable on-demand fabrication of medical instruments, ${ }^{37}$ for example, in remote areas or on the battlefield, where access to high-end medical instruments is limited.

Both metallic and polymeric materials have been used for fabrication of medical instruments. The highend AM techniques available for polymers such as stereolithography and two-photon lithography could usually achieve much higher accuracies as compared with techniques available for processing of metals. As a consequence, AM of high-end (steerable) medical instruments is currently possible only if polymeric materials are used. Some other categories of medical instruments such as many surgical guides require lower levels of accuracy and can also be manufactured from metals. On-demand fabrication of medical instruments in remote areas or in extreme conditions such as in areas hit by natural disasters or on the battlefield could only be done with low-end polymer processing techniques such as FDM and may only be realistic for the simplest medical instruments.

\section{PROSTHETICS AND ORTHOTICS}

AM has also been used for fabrication of artificial organ replacements (prosthetics) and external deformity-correcting devices (orthotics). As far as prosthetics are concerned, AM could offer easy and affordable customization options not only to make the prosthetic device fit the anatomy of the patient but also to adjust functional aspects of the prosthetic device, for 

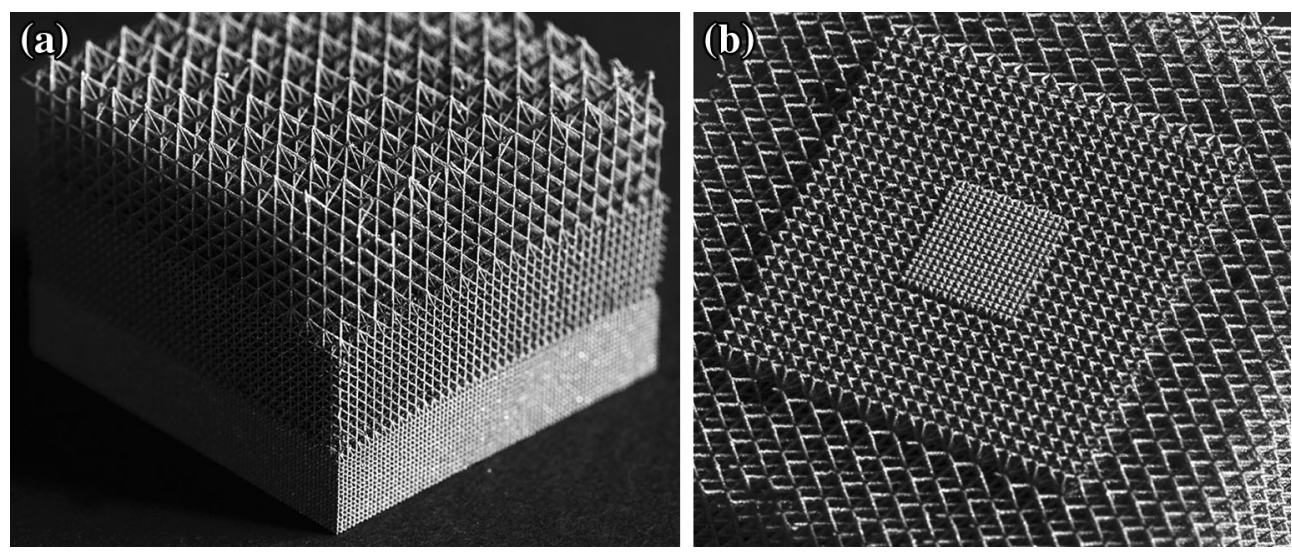

FIGURE 6. Functionally graded porous biomaterials made using selective laser melting from Ti-6Al-4V and exhibiting gradual change in porosity in the vertical (a) and diagonal (b) directions (Additive Manufacturing Laboratory, TU Delft).
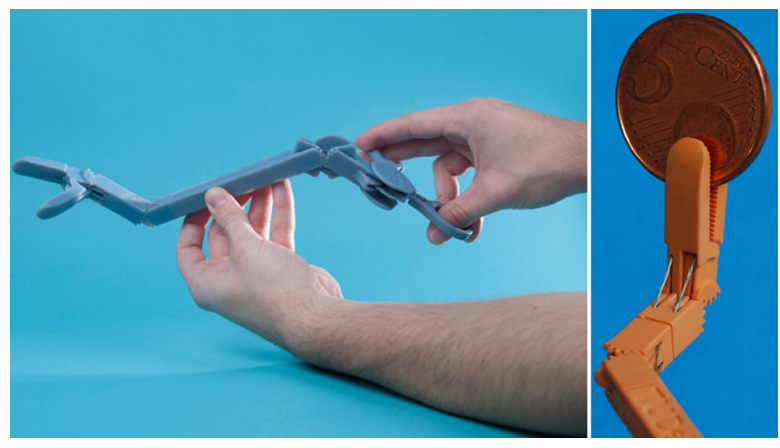

FIGURE 7. Handheld steerable laparoscopic grasping forceps (DragonFlex) designed for minimally invasive surgeries such as prostatectomy and additively manufactured from polymers using stereolithography in macro (left) and micro (right) sizes (Bio-Inspired Technology Group, TU Delft, www.bitegroup.nl, courtesy of Filip Jelínek and Paul Breedveld).

example, by modifying the dimensions of its functional parts. Depending on whether low- or high-end markets are targeted, AM could play different roles. In the high-end market, improved functionality, comfort, and esthetics could all be achieved through sophisticated AM techniques that include multimaterial and multicolor material jetting printers. In the low-end market, simple AM techniques such as FDM combined with simplified designs of prosthetics (Fig. 8) could be used to make prosthetics accessible to a larger fraction of the world population, particularly given the fact that a significant fraction of individuals requiring prosthetic devices live in war-hit low-income countries with limited access to healthcare products and services. A number of (low-cost) designs for AM prosthetic devices have been proposed in literature. ${ }^{11,29,80}$

Orthotics are no different from prosthetic devices in terms of how they could benefit from the possibilities offered by AM. ${ }^{16,52,63}$ Once more, orthotic devices could be tailored to perfectly fit the (correct) anatomy of the patient. Furthermore, materials with specific mechanical properties could be fabricated using AM using concepts similar to those used in the rational design of tissue engineering scaffolds and implants. The microarchitecture of certain parts of the orthotic device could be rationally designed so as to give rise to certain sets of desired mechanical properties; For example, AM has been used to design an ankle-foot orthosis with adjustable stiffness. ${ }^{63}$

Much research still needs to be done to fully utilize the potential of AM techniques for both the high- and low-end markets of prosthetics and orthotics. For the high-end market, advanced design and AM techniques need to be used for providing novel functionalities not currently possible in such devices, while parametric, easy-to-manufacture, and easy-to-assemble prosthetic and orthotic devices need to be developed for areas with inadequate healthcare coverage.

\section{MODELS FOR VISUALIZATION, EDUCATION, AND COMMUNICATION}

The same technology that enables image-based design and AM of implants, prosthetics, and orthotics could be used for a myriad of other purposes including communication with patients, education of medical students, visualization of malignancies, and preoperative planning. For most routine procedures, experienced clinicians can imagine anatomical features and malignancies and plan surgeries with the sole aid of two-dimensional images, not even requiring three-dimensional reconstructions of the images. The same clinicians may, however, benefit from three-dimensional reconstruction of images and physical AM models ${ }^{61,77}$ when planning complex surgeries, evaluating the suitability of certain clinical procedures for particular cases, and examining how well (patient- 


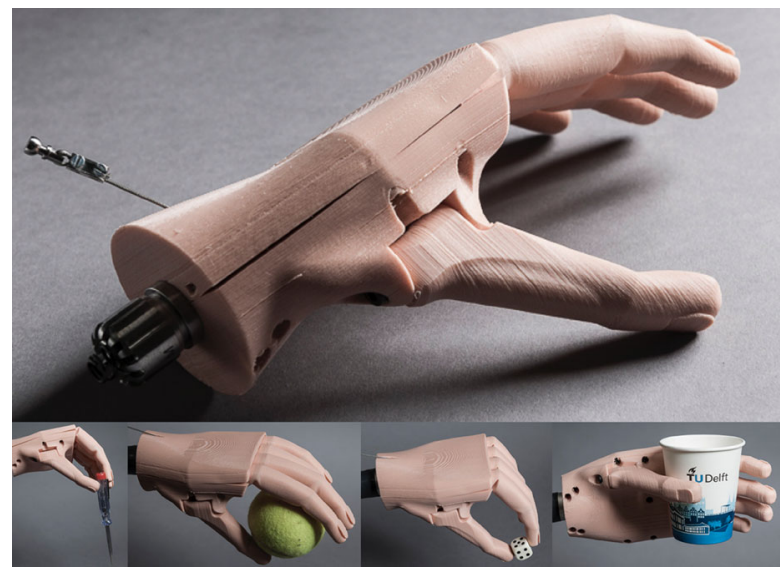

FIGURE 8. Additively manufactured prosthetic hand (FA3D hand) activated by cables and capable of holding various types of object (Bio-Inspired Technology Group, TU Delft, www.bitegroup.nl, courtesy of Jelle ten Kate, Gerwin Smit, and Paul Breedveld).

specific) implants fit the anatomy of the patient, which is also printed in the form of a physical object. The advantages might be even greater in the case of less experienced clinicians, suggesting that AM physical models could also be used as part of the learning curve of clinicians in training. ${ }^{74}$ Altogether, AM physical models might result in improved accuracy and shortened time when performing clinical procedures. Physical models might also be effective instruments when communicating with patients regarding their pathologies and the planned treatments.

Since visual aspects are of paramount importance in almost all the above-mentioned cases, multicolor and multimaterial AM techniques could be of tremendous value in fabrication of physical models aimed for visualization. Furthermore, the efficacy of physical models in improving the accuracy of clinical procedures, shortening the required time, improving the learning curve of clinicians in training, and management of patient anxiety should be thoroughly studied. There is currently not much data available to determine whether AM patient-specific physical models are actually capable of delivering the intended benefits.

\section{OTHER BIOMEDICAL AREAS}

AM has been used in other areas of biomedical research as well. An important example is the use of $\mathrm{AM}$ in fabrication of microfluidic devices that have widespread applications in biomedical research. Microfluidic devices have traditionally been made using soft lithography, which is a slow, labor-intensive, and expensive process. AM enables automated, fast, and inexpensive development of microfluidic devices, ${ }^{7,30}$ potentially from biocompatible and transparent materials that allow for both unhindered cell culture and imaging. ${ }^{65}$ Advances in AM of microfluidic devices could have important implications for areas such as organ-on-a-chip research.

Imaging-based reconstruction of pathologies and subsequent AM of colored, subject-specific demonstrative replica have been proposed as effective methods for forensic medicine, ${ }^{20}$ not only because they could help improve understanding of pathologies but also because they are excellent instruments to facilitate communication in court rooms.

Finally, pioneering works on topics such as 3D printing of bacterial communities ${ }^{15}$ continue to appear in scientific literature and promise many more applications of AM techniques in biomedical and healthcare research.

\section{CONCLUSIONS}

AM has found numerous applications in many areas of biomedical research and clinical practice. The opportunities for further application areas are only increasing in number, complexity, and added value. Some of the most important areas of application were reviewed in this editorial to lay the ground for the research and review papers appearing in this special issue. Many topics touched upon in this editorial are further expanded on in the following contributions that try to present the state of the art in this exciting area of interdisciplinary biomedical research.

\section{REFERENCES}

${ }^{1}$ Ahlfeld, T., A.R. Akkineni, Y. Förster, T. Köhler, S. Knaack, M. Gelinsky, and A. Lode. Design and fabrication of complex scaffolds for bone defect healing: combined 3D plotting of a calcium phosphate cement and a growth factor-loaded hydrogel. Ann. Biomed. Eng. 2016. doi:10.1007/s10439-016-1685-4.

${ }^{2}$ Ahmadi, S., G. Campoli, S. A. Yavari, B. Sajadi, R. Wauthlé, J. Schrooten, H. Weinans, and A. Zadpoor. Mechanical behavior of regular open-cell porous biomaterials made of diamond lattice unit cells. J. Mech. Behav. Biomed. Mater. 34:106-115, 2014.

${ }^{3}$ Amin Yavari, S., S. Ahmadi, J. van der Stok, R. Wauthlé, A. Riemslag, M. Janssen, J. Schrooten, H. Weinans, and A. A. Zadpoor. Effects of bio-functionalizing surface treatments on the mechanical behavior of open porous titanium biomaterials. J. Mech. Behav. Biomed. Mater. 36:109-119, 2014.

${ }^{4}$ Amin Yavari, S., S. Ahmadi, R. Wauthle, B. Pouran, J. Schrooten, H. Weinans, and A. Zadpoor. Relationship between unit cell type and porosity and the fatigue behavior of selective laser melted meta-biomaterials. $J$. Mech. Behav. Biomed. Mater. 43:91-100, 2015. 
${ }^{5}$ Amin Yavari, S., L. Loozen, F. L. Paganelli, S. Bakhshandeh, K. Lietaert, J. de Groot, A. C. Fluit, C. E. Boel, J. Alblas, C. Vogely, H. Weinans, and A. Zadpoor. Antibacterial behavior of additively manufactured porous titanium with nanotubular surfaces releasing silver ions. ACS Appl. Mater. Interfaces 8:17080-17089, 2016.

${ }^{6}$ Arifin, D. Y., L. Y. Lee, and C.-H. Wang. Mathematical modeling and simulation of drug release from microspheres: implications to drug delivery systems. Adv. Drug Deliv. Rev. 58:1274-1325, 2006.

${ }^{7}$ Au, A.K., W. Huynh, L.F. Horowitz, and A. Folch. 3Dprinted microfluidics. Angew. Chem. Int. Ed. Engl. 55(12):3862-3881, 2016. doi:10.1002/anie.201504382.

${ }^{8}$ Bandyopadhyay, A., F. Espana, V. K. Balla, S. Bose, Y. Ohgami, and N. M. Davies. Influence of porosity on mechanical properties and in vivo response of Ti6Al4V implants. Acta Biomater. 6:1640-1648, 2010.

${ }^{9}$ Bandyopadhyay, A., A. Shivaram, S. Tarafder, H. Sahasrabudhe, D. Banerjee, and S. Bose. In vivo response of laser processed porous titanium implants for load-bearing implants. Ann. Biomed. Eng. 2016. doi:10.1007/s10439016-1673-8.

${ }^{10}$ Bose, S., S. Tarafder, and A. Bandyopadhyay. Effect of chemistry on osteogenesis and angiogenesis towards bone tissue engineering using 3D printed scaffolds. Ann. Biomed. Eng. 2016. doi:10.1007/s10439-016-1646-y.

${ }^{11}$ Burn, M. B., A. Ta, and G. R. Gogola. Three-dimensional printing of prosthetic hands for children. J. Hand Surg. 41:e103-e109, 2016.

${ }^{12}$ Carter, S.-S.D., P.F. Costa, C. Vaquette, S. Ivanovski, D.W. Hutmacher, and J. Malda. Additive biomanufacturing: an advanced approach for periodontal tissue regeneration. Ann. Biomed. Eng. 1-11, 2016.

${ }^{13}$ Chen, Y., S. Zhou, and Q. Li. Mathematical modeling of degradation for bulk-erosive polymers: applications in tissue engineering scaffolds and drug delivery systems. Acta Biomater. 7:1140-1149, 2011.

${ }^{14}$ Chen, Y., S. Zhou, and Q. Li. Microstructure design of biodegradable scaffold and its effect on tissue regeneration. Biomaterials 32:5003-5014, 2011.

${ }^{15}$ Connell, J. L., E. T. Ritschdorff, M. Whiteley, and J. B. Shear. 3D printing of microscopic bacterial communities. Proc. Natl. Acad. Sci. 110:18380-18385, 2013.

${ }^{16}$ Creylman, V., L. Muraru, J. Pallari, H. Vertommen, and L. Peeraer. Gait assessment during the initial fitting of customized selective laser sintering ankle foot orthoses in subjects with drop foot. Prosthet. Orthot. Int. 37:132-138, 2013.

${ }^{17}$ de Damborenea, J. J., M. A. Larosa, M. A. Arenas, J. M. Hernández-López, A. L. Jardini, M. C. F. Ierardi, C. A. Zavaglia, R. Maciel Filho, and A. Conde. Functionalization of Ti6Al4V scaffolds produced by direct metal laser for biomedical applications. Mater. Des. 83:6-13, 2015.

${ }^{18}$ Do, A.-V., A. Akkouch, B. Green, I. Ozbolat, A. Debabneh, S. Geary, and A.K. Salem. Controlled and sequential delivery of fluorophores from 3D printed alginate-PLGA tubes. Ann. Biomed. Eng. 2016. doi:10.1007/s10439016-1648-9.

${ }^{19}$ Duan, B. State-of-the-art review of 3D bioprinting for cardiovascular tissue engineering. Ann. Biomed. Eng. 2016. doi:10.1007/s10439-016-1607-5.

${ }^{20}$ Ebert, L. C., M. J. Thali, and S. Ross. Getting in touch-3D printing in Forensic Imaging. Forensic Sci. Int. 211:e1-e6, 2011.
${ }^{21}$ Emons, M., K. Obata, T. Binhammer, A. Ovsianikov, B. N. Chichkov, and U. Morgner. Two-photon polymerization technique with sub-50 nm resolution by sub-10 fs laser pulses. Opt. Mater. Express 2:942-947, 2012.

${ }^{22}$ Gamsjäger, E., C. Bidan, F. Fischer, P. Fratzl, and J. Dunlop. Modelling the role of surface stress on the kinetics of tissue growth in confined geometries. Acta Biomater. 9:5531-5543, 2013.

${ }^{23}$ Gbureck, U., E. Vorndran, F. A. Müller, and J. E. Barralet. Low temperature direct 3D printed bioceramics and biocomposites as drug release matrices. J. Control. Release 122:173-180, 2007.

${ }^{24}$ Giannatsis, J., and V. Dedoussis. Additive fabrication technologies applied to medicine and health care: a review. Int. J. Adv. Manuf. Technol. 40:116-127, 2009.

${ }^{25}$ Goyanes, A., U. Det-Amornrat, J. Wang, A. W. Basit, and S. Gaisford. 3D scanning and 3D printing as innovative technologies for fabricating personalized topical drug delivery systems. J. Control. Release 234:41-48, 2016.

${ }^{26}$ Goyanes, A., P. R. Martinez, A. Buanz, A. W. Basit, and S. Gaisford. Effect of geometry on drug release from 3D printed tablets. Int. J. Pharm. 494:657-663, 2015.

${ }^{27}$ Goyanes, A., J. Wang, A. Buanz, R. Martínez-Pacheco, R. Telford, S. Gaisford, and A. W. Basit.3D printing of medicines: engineering novel oral devices with unique design and drug release characteristics. Mol. Pharm. 12:4077-4084, 2015.

${ }^{28}$ Groll, J., T. Boland, T. Blunk, J. A. Burdick, D.-W. Cho, P. D. Dalton, B. Derby, G. Forgacs, Q. Li, and V. A. Mironov. Biofabrication: reappraising the definition of an evolving field. Biofabrication 8:013001, 2016.

${ }^{29}$ He, Y., G.-H. Xue, and J.-Z. Fu. Fabrication of low cost soft tissue prostheses with the desktop 3D printer. Sci. Rep. 4:6973, 2014.

${ }^{30}$ Ho, C. M. B., S. H. Ng, K. H. H. Li, and Y.-J. Yoon. 3D printed microfluidics for biological applications. Lab Chip. 15:3627-3637, 2015.

${ }^{31}$ Hossainy, S., and S. Prabhu. A mathematical model for predicting drug release from a biodurable drug-eluting stent coating. J. Biomed. Mater. Res. A 87:487-493, 2008.

${ }^{32}$ Houben, A., J. Van Hoorick, J. Van Erps, H. Thienpont, S. Van Vlierberghe, and P. Dubruel. Indirect rapid prototyping: opening up unprecedented opportunities in scaffold design and applications. Ann. Biomed. Eng. 2016. doi: 10.1007/s10439-016-1610-x.

${ }^{33}$ Huang, W., Q. Zheng, W. Sun, H. Xu, and X. Yang. Levofloxacin implants with predefined microstructure fabricated by three-dimensional printing technique. Int. J. Pharm. 339:33-38, 2007.

${ }^{34}$ Jelínek, F., and P. Breedveld. Design for additive manufacture of fine medical instrumentation-DragonFlex case study. J. Mech. Des. 137:111416, 2015.

${ }^{35}$ Jelínek, F., R. Pessers, and P. Breedveld. DragonFlex smart steerable laparoscopic instrument. J. Med. Devices 8(1):015001, 2014.

${ }^{36}$ Kang, H.-W., S. J. Lee, I. K. Ko, C. Kengla, J. J. Yoo, and A. Atala. A 3D bioprinting system to produce human-scale tissue constructs with structural integrity. Nat. Biotechnol. 34:312-319, 2016.

${ }^{37}$ Kondor, S., C. G. Grant, P. Liacouras, M. J. R. Schmid, L. M. Parsons, V. K. Rastogi, L. S. Smith, B. Macy, B. Sabart, and $\mathrm{C}$. Macedonia. On demand additive manufacturing of a basic surgical kit. J. Med. Devices 7:030916, 2013.

${ }^{38}$ Krishnan, S., A. Dawood, R. Richards, J. Henckel, and A. Hart. A review of rapid prototyped surgical guides for 
patient-specific total knee replacement. J. Bone Joint Surg. Br. 94:1457-1461, 2012.

${ }^{39}$ Lee, V.K., and G. Dai. Printing of three-dimensional tissue analogs for regenerative medicine. Ann. Biomed. Eng. 2016. doi:10.1007/s10439-016-1613-7.

${ }^{40}$ Lin, C.-C., and A. T. Metters. Hydrogels in controlled release formulations: network design and mathematical modeling. Adv. Drug Deliv. Rev. 58:1379-1408, 2006.

${ }^{41}$ Malda, J., J. Visser, F. P. Melchels, T. Jüngst, W. E. Hennink, W. J. Dhert, J. Groll, and D. W. Hutmacher. 25th anniversary article: engineering hydrogels for biofabrication. Adv. Mater. 25:5011-5028, 2013.

${ }^{42}$ Melchels, F. P., J. Feijen, and D. W. Grijpma. A review on stereolithography and its applications in biomedical engineering. Biomaterials 31:6121-6130, 2010.

${ }^{43}$ Morris, V.B., S. Nimbalkar, M. Younesi, P. McClellan, and O. Akkus. Mechanical properties, cytocompatibility and manufacturability of chitosan:PEGDA hybrid-gel scaffolds by stereolithography. Ann. Biomed. Eng. 1-11, 2016.

${ }^{44}$ Müller, M., E. Öztürk, Ø. Arlov, P. Gatenholm, and M. Zenobi-Wong. Alginate sulfate-nanocellulose bioinks for cartilage bioprinting applications. Ann. Biomed. Eng. 1-14, 2016.

${ }^{45}$ Murphy, S. V., A. Skardal, and A. Atala. Evaluation of hydrogels for bio-printing applications. J. Biomed. Mater. Res. A 101:272-284, 2013.

${ }^{46}$ Murr, L., S. Gaytan, F. Medina, H. Lopez, E. Martinez, B. Machado, D. Hernandez, L. Martinez, M. Lopez, and R. Wicker. Next-generation biomedical implants using additive manufacturing of complex, cellular and functional mesh arrays. Philos. Trans. A Math. Phys. Eng. Sci. 368:1999-2032, 2010.

${ }^{47}$ Murr, L.E., S.M. Gaytan, E. Martinez, F. Medina, and R.B. Wicker. Next generation orthopaedic implants by additive manufacturing using electron beam melting. Int. J. Biomater. 2012:245727, 2012. doi:10.1155/2012/245727.

${ }^{48}$ Nguyen, A.K., and R.J. Narayan. Liquid-phase laser induced forward transfer for complex organic inks and tissue engineering. Ann. Biomed. Eng. 2016. doi: 10.1007/s10439-016-1617-3.

${ }^{49}$ Norman, J., R.D. Madurawe, C.M. Moore, M.A. Khan, and A. Khairuzzaman. A new chapter in pharmaceutical manufacturing: 3D-printed drug products. Adv. Drug Deliv. Rev. 2016. doi:10.1016/j.addr.2016.03.001.

${ }^{50}$ Nyberg, E.L., A.L. Farris, B.P. Hung, M. Dias, J.R. Garcia, A.H. Dorafshar, and W.L. Grayson. 3D-printing technologies for craniofacial rehabilitation, reconstruction, and regeneration. Ann. Biomed. Eng. 1-13, 2016.

${ }^{51}$ Ovsianikov, A., and B. N. Chichkov. Three-dimensional microfabrication by two-photon polymerization technique. Comput. Aided Tissue Eng. 868:311-325, 2012.

${ }^{52}$ Pallari, J. H., K. W. Dalgarno, and J. Woodburn. Mass customization of foot orthoses for rheumatoid arthritis using selective laser sintering. IEEE Trans. Biomed. Eng. 57:1750-1756, 2010.

${ }^{53}$ Park, J.H., J. Jang, J.-S. Lee, and D.-W. Cho. Three-dimensional printing of tissue/organ analogues containing living cells. Ann. Biomed. Eng. 2016. doi:10.1007/s10439016-1611-9.

${ }^{54}$ Park, S. H., D. Y. Yang, and K. S. Lee. Two-photon stereolithography for realizing ultraprecise three-dimensional nano/microdevices. Laser Photon. Rev. 3:1-11, 2009.

${ }^{55}$ Placone, J.K., J. Navarro, G.W. Laslo, M.J. Lerman, A.R. Gabard, G.J. Herendeen, E.E. Falco, S. Tomblyn, L.
Burnett, and J.P. Fisher. Development and characterization of a 3D printed, keratin-based hydrogel. Ann. Biomed. Eng. 2016. doi:10.1007/s10439-016-1621-7.

${ }^{56}$ Richards, D., J. Jia, M. Yost, R. Markwald, and Y. Mei. 3D bioprinting for vascularized tissue fabrication. Ann. Biomed. Eng. 2016. doi:10.1007/s10439-016-1653-z.

${ }^{57}$ Rumpler, M., A. Woesz, J. W. Dunlop, J. T. van Dongen, and P. Fratzl. The effect of geometry on three-dimensional tissue growth. J. R. Soc. Interface 5:1173-1180, 2008.

${ }^{58}$ Schon, B. S., G. J. Hooper, and T. B. Woodfield. Modular tissue assembly strategies for biofabrication of engineered cartilage. Ann. Biomed. Eng. 2016. doi:10.1007/ s10439-016-1609-3.

${ }^{59}$ Skardal, A., and A. Atala. Biomaterials for integration with 3-D bioprinting. Ann. Biomed. Eng. 43:730-746, 2015.

${ }^{60}$ Sobral, J. M., S. G. Caridade, R. A. Sousa, J. F. Mano, and R. L. Reis. Three-dimensional plotted scaffolds with controlled pore size gradients: effect of scaffold geometry on mechanical performance and cell seeding efficiency. Acta Biomater. 7:1009-1018, 2011.

${ }^{61}$ Spottiswoode, B., D. Van den Heever, Y. Chang, S. Engelhardt, S. Du Plessis, F. Nicolls, H. Hartzenberg, and A. Gretschel. Preoperative three-dimensional model creation of magnetic resonance brain images as a tool to assist neurosurgical planning. Stereotact. Funct. Neurosurg. 91:162-169, 2013.

${ }^{62}$ Stichler, S., T. Jungst, M. Schamel, I. Zilkowski, M. Kuhlmann, T. Böck, T. Blunk, J. Teßmar, and J. Groll. Thiol-ene clickable poly(glycidol) hydrogels for biofabrication. Ann. Biomed. Eng. 1-13, 2016.

${ }^{63}$ Telfer, S., J. Pallari, J. Munguia, K. Dalgarno, M. McGeough, and J. Woodburn. Embracing additive manufacture: implications for foot and ankle orthosis design. BMC Musculoskelet. Disord. 13:1, 2012.

${ }^{64}$ Trombetta, R., J.A. Inzana, E.M. Schwarz, S.L. Kates, and H.A. Awad. 3D printing of calcium phosphate ceramics for bone tissue engineering and drug delivery. Ann. Biomed. Eng. 2016. doi:10.1007/s10439-016-1678-3.

${ }^{65}$ Urrios, A., C. Parra-Cabrera, N. Bhattacharjee, A.M. Gonzalez-Suarez, L.G. Rigat-Brugarolas, U. Nallapatti, J. Samitier, C.A. DeForest, F. Posas, J.L. Garcia-Cordero, and A. Folch. 3D-printing of transparent bio-microfluidic devices in PEG-DA. Lab Chip 2016. doi: 10.1039/C6LC00153J.

${ }^{66}$ Vaezi, M., H. Seitz, and S. Yang. A review on 3D microadditive manufacturing technologies. Int. J. Adv. Manuf. Technol. 67:1721-1754, 2013.

${ }^{67}$ Vaithilingam, J., S. Kilsby, R. D. Goodridge, S. D. Christie, S. Edmondson, and R. J. Hague. Immobilisation of an antibacterial drug to Ti6Al4V components fabricated using selective laser melting. Appl. Surf. Sci. 314:642-654, 2014.

${ }^{68}$ Vaithilingam, J., S. Kilsby, R. D. Goodridge, S. D. Christie, S. Edmondson, and R. J. Hague. Functionalisation of Ti6Al4V components fabricated using selective laser melting with a bioactive compound. Mater. Sci. Eng. C 46:52-61, 2015.

${ }^{69}$ Van Bael, S., Y. C. Chai, S. Truscello, M. Moesen, G. Kerckhofs, H. Van Oosterwyck, J.-P. Kruth, and J. Schrooten. The effect of pore geometry on the in vitro biological behavior of human periosteum-derived cells seeded on selective laser-melted Ti6A14V bone scaffolds. Acta Biomater. 8:2824-2834, 2012.

${ }^{70}$ van der Stok, J., M. Koolen, M. de Maat, S. AMin Yavari, J. Alblas, P. Patka, J. Verhaar, E. van Lieshout, A. A. Zadpoor, and H. Weinans. Full regeneration of segmental 
bone defects using porous titanium implants loaded with BMP-2 containing fibrin gels. Eur. Cells Mater. 2015:141154, 2015.

${ }^{71}$ Vanderburgh, J., J.A. Sterling, and S.A. Guelcher. 3D printing of tissue engineered constructs for in vitro modeling of disease progression and drug screening. Ann. Biomed. Eng. 2016. doi:10.1007/s10439-016-1640-4.

${ }^{72}$ Wang, C., Z. Tang, Y. Zhao, R. Yao, L. Li, and W. Sun. Three-dimensional in vitro cancer models: a short review. Biofabrication 6:022001, 2014.

${ }^{73}$ Wang, X., S. Xu, S. Zhou, W. Xu, M. Leary, P. Choong, M. Qian, M. Brandt, and Y. M. Xie. Topological design and additive manufacturing of porous metals for bone scaffolds and orthopaedic implants: a review. Biomaterials 83:127-141, 2016.

${ }^{74}$ Waran, V., V. Narayanan, R. Karuppiah, S. L. Owen, and T. Aziz. Utility of multimaterial 3D printers in creating models with pathological entities to enhance the training experience of neurosurgeons: technical note. J. Neurosurg. 120:489-492, 2014.

${ }^{75}$ Zadpoor, A. A. Bone tissue regeneration: the role of scaffold geometry. Biomater. Sci. 3:231-245, 2015.

${ }^{76}$ Zadpoor, A.A. Mechanical meta-materials. Mater. Horiz. 3:371-381, 2016. doi:10.1039/C6MH00065G.

${ }^{77}$ Zein, N. N., I. A. Hanouneh, P. D. Bishop, M. Samaan, B. Eghtesad, C. Quintini, C. Miller, L. Yerian, and R. Klatte. Three-dimensional print of a liver for preoperative planning in living donor liver transplantation. Liver Transplant. 19:1304-1310, 2013.

${ }^{78}$ Zhang, Q., Y. Jiang, Y. Zhang, Z. Ye, W. Tan, and M. Lang. Effect of porosity on long-term degradation of poly
( $\varepsilon$-caprolactone) scaffolds and their cellular response. Polym. Degrad. Stab. 98:209-218, 2013.

${ }^{79}$ Zhang, Y.S., K. Yue, J. Aleman, K. MollazadehMoghaddam, S.M. Bakht, J. Yang, W. Jia, V. Dell'Erba, P. Assawes, and S.R. Shin. 3D bioprinting for tissue and organ fabrication. Ann. Biomed. Eng. 1-16, 2016.

${ }^{80}$ Zuniga, J., D. Katsavelis, J. Peck, J. Stollberg, M. Petrykowski, A. Carson, and C. Fernandez. Cyborg beast: a low-cost 3d-printed prosthetic hand for children with upper-limb differences. BMC Res. Notes 8:1, 2015.

\section{AMir A. ZADPOOR}

Additive Manufacturing Laboratory, Department of Biomechanical Engineering

Delft University of Technology (TU Delft), Mekelweg 2, Delft 2628 CD, The Netherlands Electronic mail: a.a.zadpoor@tudelft.nl

Jos MALDA

Department of Orthopaedics, University Medical Center Utrecht, PO Box 85500, 3508 GA Utrecht, The Netherlands Department of Equine Sciences, Faculty of Veterinary Medicine, Utrecht University, Yalelaan 112, 3584 CM Utrecht, The Netherlands 